Rabaska

Revue d'ethnologie de l'Amérique française

\title{
Marius Barbeau et la chanson traditionnelle française : évolution d'un héritage
}

\section{Marcel Bénéteau}

Volume 13, 2015

Présence de Marius Barbeau : l'invention du terrain en Amérique

française. Autour d'un legs centenaire (1914-2014)

URI : https://id.erudit.org/iderudit/1033753ar

DOI : https://doi.org/10.7202/1033753ar

Aller au sommaire du numéro

Éditeur(s)

Société québécoise d'ethnologie

ISSN

1703-7433 (imprimé)

1916-7350 (numérique)

Découvrir la revue

Citer cet article

Bénéteau, M. (2015). Marius Barbeau et la chanson traditionnelle française :

évolution d'un héritage. Rabaska, 13, 98-111. https://doi.org/10.7202/1033753ar 


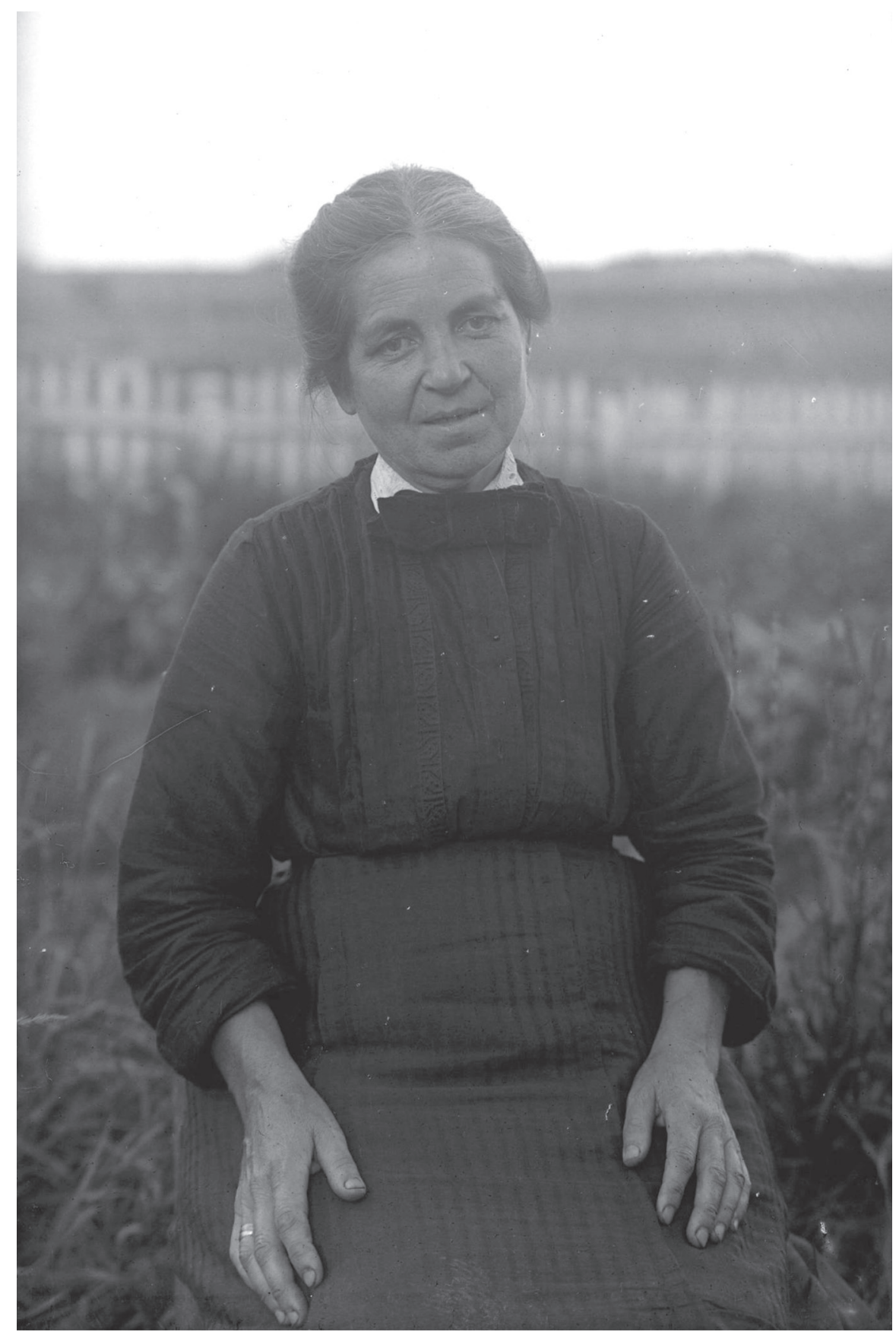

Madame Jean Bouchard, chanteuse folklorique,

Les Éboulements, Québec, 1916

Photo de Marius Barbeau

Source : Musée canadien de l'histoire nº 37265. 


\section{Marius Barbeau et la chanson traditionnelle française : évolution d'un héritage}

Marcel BÉnÉteAu

Université de Sudbury

Dans un article récent, Daniel Guilbert affirme que Marius Barbeau est « reconnu aujourd'hui comme le chercheur qui est à la source de l'intérêt qu'on porte maintenant à la chanson traditionnelle au Québec ${ }^{1} »-$ et, par extension, dans l'ensemble du Canada français. Ce n'est pas mon intention ici de diminuer l'influence qu'on accorde à Marius Barbeau dans la sauvegarde et la mise en valeur du répertoire de l'Amérique française ou de nier l'importance de son œuvre dans les mondes scientifique et artistique. Je propose cependant de passer sous la lentille sa conception de la chanson traditionnelle, ainsi que son approche de la collecte, de la conservation et de la diffusion du répertoire canadien-français afin de circonscrire l'effet que ses travaux ont eu sur les collectes et les collecteurs subséquents, et aussi sur la place qu'occupe aujourd'hui la chanson traditionnelle dans le champ des études ethnologiques du Canada français.

En parlant de l'influence de Barbeau sur les folkloristes, je me permets une petite parenthèse personnelle. Je dois sans aucun doute me compter parmi ceux et celles qui ont été inspirés et guidés par ce grand pionnier. Comme jeune chercheur, amateur et largement autodidacte, les trois volumes de son « Répertoire de la chanson folklorique française au Canada »-Le rossignol $y$ chante, En roulant ma boule et Le roi boit ${ }^{2}$ - ont été parmi mes premiers guides de terrain lors de mes enquêtes dans le sud-ouest ontarien. Grâce à la lecture de ses œuvres, je me suis lancé dans la cueillette, imbu d'un sens de

1. Daniel Guilbert, «La Légende des "Veillées du bon vieux temps" (2 ${ }^{\text {ème }}$ partie) », Bulletin Mnémo, vol. 13, $\mathrm{n}^{\circ}$ 1, 2011, http://www.mnemo.qc.ca/spip/bulletin-mnemo/article/la-legende-desveillees-du-bon, consulté le 10 septembre 2015.

2. Marius Barbeau, Le rossignol y chante. Première partie du répertoire de la chanson folklorique française au Canada, Ottawa, Musée national de l'Homme, Musées nationaux du Canada, 1979, 485 p. ; En roulant ma boule. Deuxième partie du répertoire de la chanson folklorique française au Canada, Ottawa, Musée National de l'Homme, Musées nationaux du Canada, 1982, 753 p.; Le roi boit. Troisième partie du répertoire de la chanson folklorique française au Canada, Ottawa, Musée National de l'Homme, Musées nationaux du Canada, 1987, 623 p. 
mission pour la sauvegarde du folklore de mon petit coin de pays, un folklore qui, lui aussi, « allait bientôt s'endormir de son dernier sommeil " ». La tâche urgeait : les plus jeunes de mes informateurs étaient octogénaires et la plupart n'avaient pas chanté leurs chansons depuis une cinquantaine d'années. Je convenais aussi avec Barbeau qu'en étant ainsi sauvés de l'oubli, ces objets précieux seraient inévitablement repris par les nouvelles générations - et à ma grande satisfaction, plusieurs des chansons que j'ai recueillies et enregistrées ont effectivement été par la suite réinterprétées par des artistes français et québécois, populaires aussi bien que classiques. Comme Marius Barbeau, j'ai dédaigné tout ce qui n'était pas folklore traditionnel, authentique, amené par nos ancêtres de la France et vaguement médiéval - pour ne pas dire tout ce qui avait été appris à l'école et diffusé par l'abbé Gadbois"; surtout ne fallait-il pas que le répertoire recueilli soit contaminé par toute pièce pouvant être identifiée comme « littéraire ». À vrai dire, je n'avais pas le temps de m'attarder sur ce que les gens chantaient - c'est-à-dire sur le répertoire tel qu'il existe actuellement -, ce qu'ils avaient choisi et façonné pour répondre au contexte particulier de leur existence à la frontière même de la francophonie. Il fallait aller droit au but : recueillir ce qui restait d'une culture authentiquement française sur les bords - non pas du Saint-Laurent, dans mon cas - mais de la rivière Détroit, la plus ancienne colonie française à l'ouest de Montréal : se hâter de redonner à la population une culture qu'elle ne savait même pas avoir possédée. Je me suis même plu à dire que certaines chansons avaient été mieux conservées sur les bords de la rivière Détroit que le long du fleuve Saint-Laurent.

Évidemment, je caricature, jusqu'à un certain point, mais seulement pour démontrer comment, soixante-quinze ans après les enquêtes de Barbeau et à plus de mille kilomètres des terrains qu'il avait parcourus, sa conception de la chanson traditionnelle demeurait séduisante et convaincante. Mon cheminement et ma formation éventuelle m'ont mené à adopter une perspective plus complexe et plus nuancée envers le patrimoine immatériel et aussi à constater que la pensée de Barbeau lui-même était beaucoup plus subtile que je ne l'avais imaginée. Sa vision du folklore a été très complexe, comme le soulignent plusieurs intervenants dans ce colloque, et elle n'a cessé d'évoluer tout au cours de sa vie. Mais il reste que l'ancienne chanson française occupe une place privilégiée dans le firmament folklorique de Barbeau et que la perception qu'il en a semble demeurer relativement constante. Il est donc pertinent, considérant la place d'honneur qu'on accorde

3. Sort que prévoit Barbeau pour la chanson Le Berger colin, qu'il avait recueillie sur les bords du Saint-Laurent (Le rossignol y chante, p. 75); chanson pourtant toujours vivante en Ontario 75 ans plus tard (coll. Marcel Bénéteau 202.11a).

4. Charles-Émile Gadbois, éditeur de la célèbre série de La Bonne chanson, Saint-Hyacinthe, Éditions de la Bonne Chanson, 1938-1954, 10 vol. 
à Barbeau dans le domaine de la chanson traditionnelle au Canada français, d'examiner certaines des notions qu'il avançait à propos de cette composante du patrimoine et de considérer l'influence que ses notions ont exercée sur le répertoire, son étude et sa diffusion. Comment Barbeau voit-il cet élément clé du folklore - sa nature, ses caractéristiques, mais aussi son rôle et sa fonction sociale? Qu'est-ce qui est, pour lui, une chanson traditionnelle ?

\section{Conception de la chanson traditionnelle}

Avant tout, Barbeau voit la chanson traditionnelle comme le produit d'un âge complètement divorcé de la vie moderne, un monde qui n'a rien à voir avec le temps actuel. Serge Gauthier, dans sa discussion de l'invention du terrain folklorique en Charlevoix ${ }^{5}$, va droit au cour de la conception du folklore de Barbeau. Selon lui, celle-ci relève en grande partie d'une vision idyllique de la société du Moyen Âge, période qui représente le mieux les traditions qu'il veut recueillir 6 . Gauthier maintient d'ailleurs que les disciples les plus importants de Barbeau, Luc Lacourcière et Félix-Antoine Savard, vont à leur tour « retenir le cadre médiéval comme étant aussi la période où les traditions françaises trouvaient leur âge d'or ${ }^{7}$ », perspective qui va donc s'ancrer fermement dans les études subséquentes. Dans le domaine de la chanson, ce sont certes des thèmes médiévaux - princesses dans des tours, légendes miraculeuses, chevaliers à la claire épée - qui suscitent les commentaires les plus enthousiastes de Barbeau. On n'a qu'à se rappeler les commentaires qu'il fait au sujet de ses informateurs les plus doués pour constater la distance qu'il perçoit entre ce répertoire transmis depuis la nuit des temps et les temps modernes. Au sujet de Gilbert Marin, Barbeau proclame : «Il faisait penser à un jongleur tombé des pages enluminées de quelque parchemin moyenâgeux ${ }^{8}$ ». Pour Madame Jean « Français » Bouchard, il écrit : « Mon émerveillement à l'audition de mélodies modales était la même que l'on éprouve devant les vieux temples gothiques de la Normandie - ceux de Caen ou de Bayeux, où elles prirent naissance »; et encore : "Cette architecture grandit encore dans la nuit lumineuse, au clair de la lune ; de même la voix faible d'une chanteuse aux cheveux blanchissants enlumine d'un rayon pâle les personnages de ces chansons qui sont comme des ombres de pierre ou des profils légendaires ${ }^{9} »$. Au sujet de Vincent Ferrier de Repentigny, le grand informateur d'É.-Z. Massicotte, Barbeau affirme que : « Les raffinements de son style vocal donnent l'illusion d'une formation archaïque, dans laquelle

5. Serge Gauthier, Charlevoix ou la création d'une région folklorique. Étude du discours de folkloristes québécois (1916-1930), Québec, Presses de l’Université Laval, 2006, 208 p.

6. Ibid., p. 80.

7. Loc. cit.

8. Marius Barbeau, Le Romancero du Canada, Montréal, Éditions Beauchemin, 1937, p. 128.

9. Ibid., p. 160-161. 
il y a de l'atavisme, ou plutôt, un retour émouvant vers l'âge éloigné de l'Ancienne France ${ }^{10} »$.

Ces élans poétiques n'ont rien de faux ou de sinistre en soi. Nul ne peut nier la survivance de pièces et de formes musicales anciennes dans le répertoire traditionnel. Mais, pour Barbeau, cette généalogie semble être la principale marque d'authenticité qui définit les chansons traditionnelles en Amérique française et qui confirme leur valeur. Et ces informateurs ne sont pas choisis au hasard: dans le résumé d'un cours donné à la faculté des lettres de l'Université Laval en 1945, Barbeau affirme que l'informateur idéal est « l'homme rustique, l'illettré, le sauvage ${ }^{11}$. » Dans les commentaires de Serge Gauthier, " cet informateur "sauvage" est hors du temps et conçu immaculé ou pur. Il n'est pas entaché par le monde moderne ${ }^{12} »$. Dans les mots mêmes de Barbeau : «Un chanteur de chansons populaires qui a quelque instruction de la ville, n'est pas d'ordinaire le meilleur. Il a pris ses connaissances un peu partout; il s'y trouve du bon et du mauvais; il faut de l'expérience pour démêler l'un de l'autre ${ }^{13}$ ».

Ce n'est pas par hasard que l'œuvre maîtresse de Barbeau ait prit comme titre le Romancero du Canada. S'inspirant du modèle de Georges Doncieux en France, il fait le lien explicite avec une tradition ancienne et bien à l'écart du monde moderne, " romancero » étant un terme espagnol désignant à l'origine une collection de romances ou de récits versifiés au Moyen Âge. On voit davantage cet engouement dans certains autres titres de Barbeau, comme par exemple Le Québec, où survit l'ancienne France ${ }^{14}$ ou bien Jongleur Songs of Old Québec ${ }^{15}$, ainsi que dans les divisions thématiques du recueil Le rossignol y chante, premier volume de son répertoire de la chanson folklorique française au Canada, le seul publié de son vivant : « Complaintes du moyen âge »; " Princesses emprisonnées dans une tour »; "Anciennes chansons »; " Chants religieux et miracles »; « Nocturnes et aubades »; « Pastourelles ».

Évidemment ce ne sont pas des thèmes inventés ou imaginés par Barbeau; ils sont bel et bien présents dans le répertoire et la décision de leur donner la place d'honneur dans un recueil est un choix tout à fait justifiable. Mais il est instructif de mettre en contraste le jugement que porte Barbeau sur

10. Ibid., p. 152.

11. Marius Barbeau, "En quête de connaissances anthropologiques et folkloriques dans l'Amérique du Nord depuis 1911 ». Résumé d'un cours donné à la Faculté des lettres, mars-octobre 1945, Québec, Archives de folklore de l'Université Laval, 1945, 82 p. (manuscrit dactylographié).

12. Serge Gauthier, op.cit., p. 54.

13. Marius Barbeau, loc. cit.

14. Marius Barbeau, Québec où survit l'ancienne France, Québec, Librairie Garneau, Limitée, $1937,175 \mathrm{p}$.

15. Marius Barbeau, Jongleur Songs of Old Québec, traduit en anglais par Harold Boulton et Ernest MacMillan, New Jersey/Toronto, Rutgers University Press/Ryerson Press, 1962, 202 p. 
les chansons composées sur le terrain d'Amérique qui font aussi partie du répertoire folklorique. Selon lui, « ces manifestations d'art rustique, toutes intéressantes qu'elles puissent être, sont dépourvues de style autant que de grammaire ${ }^{16} »$. Déjà en 1919, il expliquait dans le Journal of American Folklore qu' " Il faut établir une distinction entre les pièces qui viennent de France et celles - relativement peu nombreuses - qui furent composées au Canada [...]. Tandis que la plupart de ces chants venus de France prirent naissance à une époque où les jongleurs (chanteurs ou compositeurs populaires) possédaient encore une éducation artistique particulière, les compositions canadiennes, elles, sortent surtout de la classe des illettrés, ce qui explique sans doute la différence assez sensible qu'on remarque dans leur valeur littéraire ${ }^{17} \gg$.

Il serait peut-être trop facile ici de souligner les contradictions implicites et explicites - l'instruction livresque, par exemple, paraissant tout à coup supérieure à la sagesse infuse de l'homme sauvage - mais le point à retenir, je crois, c'est que les Canadiens français ne sont pas des créateurs de folklore, bien qu'ils en soient les transmetteurs par excellence. Sous-entendue dans cette perspective, l'idée que le folklore lui-même n'est que transmis et non créé ou recréé. Et c'est le rôle du folkloriste de retrouver ceux et celles qui le transmettront le plus fidèlement. Bien que cette approche ait une certaine valeur - et ait eu comme résultat non négligeable la sauvegarde des plus anciennes traditions orales européennes en Amérique du Nord - il me semble quand même qu'au tout début de la collecte scientifique des faits folkloriques au Canada français, une telle attitude ne pouvait que fausser en quelque sorte le portrait du répertoire de chansons chantées par la population, d'abord en limitant les genres de chansons dignes de collecte et aussi en excluant tout un pan de la population - la population instruite et urbaine - susceptibles de pouvoir apporter des contributions importantes à nos connaissances.

En examinant le rôle de Barbeau dans la diffusion de la chanson traditionnelle dans le cadre des Veillées du bon vieux temps, Daniel Guilbert propose que « à l'aube de l'histoire médiatique de la musique traditionnelle, le folklore est déjà perçu comme étant quelque chose de l'ancien temps et non comme un processus vivant qui se transforme selon les contextes socio-historiques et politiques qui les entourent ${ }^{18} »$. Guilbert identifie bien la conception du folklore de Barbeau comme un produit et non comme une pratique. Évidemment, cette vision était courante chez les folkloristes de l'époque ; et puisque, de toute évidence, le produit en question était en

16. Marius Barbeau, Romancero du Canada, op. cit., p. 184.

17. Marius Barbeau, « Chants populaires du Canada, recueillis par É.-Z. Massicotte et préparés par C-M. Barbeau », dans Journal of American Folklore, vol. XXXII, n ${ }^{\circ}$ CXXIII, janvier-mars 1919, p. 7.

18. Daniel Guilbert, "La Légende des "Veillées du bon vieux temps" (1 $1^{\text {ere }}$ partie) », Bulletin Mnémo, vol. 12, $\mathrm{n}^{\circ}$ 4, 1910, http://www.mnemo.qc.ca/spip/bulletin-mnemo/article/la-legende-desveillees-du-bon, consulté le 10 septembre 2015. 
train de disparaître, face à la modernité, ces derniers voyaient leur mission comme celle de la sauvegarde d'un héritage, de sa valorisation et même de sa restauration. Le folklore est un legs précieux qui doit être protégé : selon cette perspective, toute influence externe, toute rencontre interculturelle, tout contact avec la modernité aura un effet néfaste. Toute évolution - si ce n'est que celle inspirée par l'esprit des Canadiens représentés par les voyageurs, les habitants et les bûcherons - ne peut que mener à la dégradation. Le monde moderne, avec son ascendance incontournable, est l'ennemi de cette ancienne tradition française et finira par la détruire. D'où le besoin de la sauvegarder avant qu'elle ne s'éteigne à jamais. D'où aussi la nécessité - qui sera discutée un peu plus tard - de la restaurer en recréant des versions « critiques 》 des chansons, de retrouver leurs formes originales.

Nous savons bien que les attitudes de Barbeau lui-même ont suivi une évolution complexe à cet égard et que, dans d'autres contextes, il célèbre l'aspect vivant de la tradition, les transferts culturels et la fusion de traditions pour créer une identité nationale ${ }^{19}$. Mais il est évident que ses premiers écrits sur la chanson ont eu une influence considérable chez les folkloristes qui ont suivi le chemin qu'il a ouvert. Jean-Pierre Pichette démontre bien l'influence du Romancero sur des chercheurs comme Luc Lacourcière, Germain Lemieux, Anselme Chiasson et Conrad Laforte ${ }^{20}$. On retrouve certainement l'empreinte de Marius Barbeau dans les travaux de Germain Lemieux - qui reconnut que « ma vocation de folkloriste avait commencé le jour où j'ai lu le Romancero de Barbeau ${ }^{21}$ ». Si Barbeau publie en 1962 Jongleur Songs of Old Québec, Lemieux dix ans plus tard intitule son étude hommage aux conteurs franco-ontariens Les Jongleurs du billochet, disant que « le terme "jongleur" a passablement évolué au cours des siècles ; nous le prenons ici dans son sens médiéval d'amuseur ou de diseur de fabliaux ou de $\operatorname{contes}^{22}$ ». Comme Louis-Martin Savard l'a démontré au cours de ce colloque, Barbeau a aussi grandement influencé Joseph-Thomas LeBlanc dans le choix et la présentation des chansons acadiennes. Pour les générations de chercheurs qui suivront ses pas, c'est évidemment Barbeau qui donna le ton pour la chanson traditionnelle.

Mais l'influence de Barbeau ne se limite pas au monde scientifique. Sa vision de la chanson folklorique va bien au-delà de son étude universitaire

19. Voir par exemple Christine Bricault, Anne-Marie Desdouits et Dominique Sarny, « Rétrospective de la discipline. La Conception du folklore de trois pionniers : Marius Barbeau, Luc Lacourcière et Carmen Roy », Ethnologies, vol. 26, n 2, 2004, p. 21-56.

20. Jean-Pierre Pichette, «Le Chercheur de trésors ou l'influence d'un livre : Marius Barbeau et le Romancero du Canada », dans Cahiers Charlevoix 7. Études franco-ontariennes, Sudbury, Société Charlevoix et Éditions Prise de parole, 2006, p. 85-141 ; voir surtout p. 131-137.

21. Cité par Jean-Pierre Pichette, ibid., p. 133.

22. Germain Lemieux, Les Jongleurs du billochet : conteurs et contes franco-ontariens, Montréal, Éditions Bellarmin, 1972, p. 12. 
pour englober sa valeur utilitaire et sa mise en scène artistique. Pour bien comprendre l'influence de Barbeau sur la place qu'occupe aujourd'hui la chanson traditionnelle dans la pensée ethnologique au Canada français, il faut se pencher sur ces autres aspects de sa vision.

\section{Utilité et rôle de la chanson}

Si Barbeau se donne la mission de trouver et de documenter les traces de l'Ancienne France parmi le peuple rural québécois et canadien-français, c'est avant tout au service d'une idéologie. La démarche s'inscrit parmi celles d'une longue lignée d'opposants au gouverneur Durham qui avait rudement qualifié les Canadiens de peuple sans histoire et sans littérature. L'œuvre de Barbeau permet de reconnaître cette culture invisible en faisant découvrir ses racines. Serge Gauthier compare l'approche de Barbeau au concept proposé par Hobsbawn de la «tradition inventée » pour « cimenter et justifier » la nation par un recours au passé : « Les traits culturels sont ici des éléments fondateurs de la tradition nationale du nouvel État-nation : langue, pratiques populaires, chants, danses, costumes, contes, légendes [...] l'objectif étant de former une tradition nationale qui constitue, unifie et justifie l'État-nation ${ }^{23}$. » Le folklore sert alors de matière brute pour forger une identité culturelle. Contrairement à ses contemporains, la nation qu'envisage Barbeau ne se limite pas à la nation québécoise ou même canadienne-française : c'est le Canada entier avec ses deux peuples fondateurs qui constitue la "nation » de Barbeau et le folklore a un rôle important à jouer dans la réalisation de ce projet : « Le fruit de la connaissance de notre folklore sera notre indépendance nationale, la floraison de notre légitime fierté, le bien-être, le bonheur de tous dans une culture nationale qui s'acheminera en l'an mil ${ }^{24} \gg$. Et parmi tous les éléments culturels traditionnels, ce sont les chansons - véhiculées par le langage universel de la musique - qui réussiront le mieux à inspirer les artistes, à aplanir les classes sociales, à jeter des ponts entre les deux solitudes.

Pour ce faire, Barbeau constate qu'il lui faut sortir du monde scientifique et se lancer dans la mise en scène des chansons et leur diffusion auprès du grand public. Selon Daniel Guilbert, on retrouve, dès les premières initiatives de Barbeau dans ce domaine, tous les éléments caractéristiques de son approche. Il s'agit des Veillées du bon vieux temps, deux soirées aujourd'hui célèbres organisées par Barbeau à la bibliothèque Saint-Sulpice de Montréal en 1919. Guilbert analyse l'influence de ces soirées sur le développement de la chanson traditionnelle sur la scène musicale au Canada français. Selon lui, le but fondamental des soirées était « clairement indiqué par Barbeau dans la Préface du livre qui a suivi : "de signaler les thèmes mélodiques du terroir

23. Serge Gauthier, op. cit., p. 6.

24. Christine Bricault, Anne-Marie Desdouits et Dominique Sarny, op.cit., p. 27. 
canadien à nos compositeurs" de musique classique (la haute culture), et non pour promouvoir des artistes ruraux, ou leur musique, à l'état brut et donc indigeste, à l'élite culturelle de la ville. ${ }^{25} »$ Ou dans les paroles de Barbeau lui-même : «Le moment n'était-il pas venu de s'attaquer aux préjugés urbains en faisant connaître, par moyen d'exemples, - et par la suite aimer - ce patrimoine obscur que la population rurale conserve inconsciemment pour la régénération de la race $^{26}$ ?»

Barbeau rappelle avec fierté les retombées de cette initiative dans son livre Québec où survit l'Ancienne France :

On venait d'interpréter, au programme [d'un des Festivals] et à la radio, des compositions originales dont le thème était des chansons et des airs de danse. Plusieurs de ces compositions ont, depuis, été publiées et vulgarisées au Canada et à l'étranger. Les chants de notre pays peuvent faire naître de grandes œuvres, comme d'autres chants en ont inspirées en Europe. Nulle part ils ne sont plus nombreux, plus variés et plus admirables qu'au Canada ; ils y seront peut-être appelés à produire une renaissance du goût en musique et un renouveau dans l'art. ${ }^{27}$

Il est bien connu que de nombreux musiciens et artistes classiques ont effectivement répondu au défi lancé par Barbeau, collaborant avec lui dans les années suivantes pour créer cette nouvelle tradition artistique : on note, entre autres, des deux côtés de la frontière linguistique, des musiciens et artistes comme Ernest MacMillan, Alfred Laliberté, Achille Fortier, Claude Champagne, Hector Gratton, Oscar O’Brien, Léo-Pol Morin, Léo Roy, Irvin Cooper. Mais Barbeau n'est pas ouvert à toutes les productions sur scène. Guilbert souligne l'écart entre la présentation du folklore promue par Barbeau et celle des musiciens populaires comme Conrad Gauthier qui lui aussi commencera deux ans plus tard à organiser ses propres « Veillées du bon vieux temps ». Le fait que ces nouvelles productions reflètent peut-être un style qui se rapproche davantage des goûts du «peuple » ne semble pas pencher en leur faveur. Guilbert cite à cet égard Luc Lacourcière qui défend l'entreprise de Barbeau en critiquant ces nouvelles soirées de musique comme des « pastiches de la paysannerie » pour avoir osé présenter la chanson folklorique de façon plus contemporaine et commerciale :

C'est à partir de 1920, en effet, que des amateurs chez qui on ne retrouvait rien du folklore ni de l'art, usurpant le titre même des soirées de Saint-Sulpice, entreprirent d'improviser des « veillées du bon vieux temps ». On s'y contentait de mimer notre paysannerie. Le genre La Débauche envahit les tréteaux ; il

25. Daniel Guilbert, « La Légende des "Veillées du bon vieux temps" (1 1 iere partie), op. cit.

26. Marius Barbeau, Veillées du bon vieux temps à la bibliothèque Saint-Sulpice de Montréal, les 18 mars et 24 avril 1919. Sous les auspices la Société historique de Montréal et de la Société de folklore d'Amérique (Section de Québec), Montréal, G, Ducharme, libraire-éditeur, 1920, p. 2.

27. Marius Barbeau, Québec où survit l'ancienne France, op. cit., p. 116. 
triomphe encore aujourd'hui dans certains programmes radiophoniques. Ces caricatures intempestives faussèrent la notion même d'une science qui venait de naitre. ${ }^{28}$

Guilbert souligne cependant que les soirées organisées par Barbeau constituaient aussi « un pastiche, ou encore plus précisément une appropriation, savante ou non, et "authentique" ou non, de la tradition. ${ }^{29} \gg$ Il est difficile de ne pas arriver à la même conclusion en lisant la description des soirées originales, de la plume de Barbeau lui-même :

«C'est comme dans le bon vieux temps! » disait-on de toutes parts. Même avant que le chanteur de Repentigny - vêtu en bûcheron, portant grand chapeau de feutre relevé, ceinture fléchée et bottes de draveur eût soulevé l'enthousiasme avec sa chanson de rames « Envoyons de l'avant, nos gens ! » nous n'avions pas de doute sur le sort de notre entreprise. L'auditoire semblait gagné. Violoneux, $[\ldots]$ danseurs, conteurs se suivirent pendant plus de deux heures sans qu'on se fatiguât de les applaudir. [...] Le fond de scène, ingénieusement préparé par M. Émile Vaillancourt, représentait l'intérieur d'une maison rustique, avec "banclit", chaises canadiennes, huche, baratte, rouet "à canelle", lampe à bec, fanal de ferblanc, banc des seaux, fusil "à pierre", “jougs" de porteur d'eau, moule à cuillers, ceinture fléchée et catalognes... On était même venu de loin pour assister à cette résurrection du passé. ${ }^{30}$

C'est cette tradition, " pure et authentique », que Barbeau veut conserver, restaurer et promouvoir. Non seulement les chansons recueillies doivent être pures et non contaminées, mais elles doivent être présentées de façon authentique. Le mot « authentique » paraît, à première vue, être un thème neutre, mais Barbeau lui donne un sens particulier : les chansons doivent être présentées de façon « raffinée » pour en faire ressortir la beauté - comme si elles seraient passées inaperçues ou indignes si appréhendées par la classe artistique dans leur forme brute.

Certes nous connaissons la rudesse, la naïveté, et même les âpretés de cet art robuste des gens du pays. [...] Notre seule appréhension venait de ce que l'auditoire pût se laisser distraire par des trivialités. Il arrive souvent qu'un mot mal venu choque l'oreille susceptible, qu'un geste ou qu'une fioriture ne soit pas conforme aux préceptes du conservatoire ou des professeurs d'opéra. ${ }^{31}$

Les efforts encourus pour éviter ce genre de faux-pas relève presque de la pure comédie :

28. Daniel Guilbert, «La Légende des "Veillées du bon vieux temps" (3 $3^{\text {ième }}$ partie), Bulletin Mnémo, vol. 13, $\mathrm{n}^{\circ}$ 4, 2011, http://www.mnemo.qc.ca/spip/bulletin-mnemo/article/la-legende-desveillees-du-bon, consulté le 10 septembre 2015.

29. Loc. cit.

30. Marius Barbeau, Veillées du bon vieux temps, op. cit., p. 3.

31. Ibid., p. 2. 
Afin de signaler les thèmes mélodiques du terroir canadien à nos compositeurs, nous voulions faire exécuter sur instruments quelques rhapsodies ou chansons fondées sur des airs du pays. Après avoir cherché en vain, notre choix dut s'arrêter à des variations banales pour piano, composées il y a un demi-siècle par un Allemand en voyage ! Où étaient donc les compositeurs canadiens ? Moins dépourvus de chansons populaires harmonisées - bien que fort peu soient au point - nous ne pûmes trouver d'interprète possédant la technique spéciale requise $[\ldots]$. Deux chanteuses - mieux habituées au grand opéra - nous firent faux bond au dernier moment. Mesdemoiselles Fisher et Montet voulurent bien, à l'improviste, combler cette lacune en lisant quelques chansons publiées avec accompagnement par MM. Achille Fortier et Amédée Tremblay. Le concours gracieux de Miss Wyman, à la seconde soirée de folklore, fit clairement saisir la valeur artistique de la chanson populaire. Un critique écrivait : [...] C'est en entendant Mlle Loraine Wyman dire si admirablement des chansons françaises et des chansons canadiennes qu'on pu constater comment, d'une chose fruste, la chanson populaire s'élève aux sommets de l'art avec, pourtant, les mêmes éléments de texte et de musique ${ }^{32}$.

Il est un peu ironique que, sous cet aspect, Barbeau ne soit pas tellement loin des propos de l'abbé Burque, qui, dans son Nouveau Chansonnier canadienfrançais $^{33}$, remanie des chansons à gauche et à droite pour les purger de tout caractère grossier, rustique - chansons infectées par ce qu'il nomme « la maladie folklorique ${ }^{34} \gg$. On ne peut comparer ici ni la méthode, ni l'idéologie, ni les intentions des deux hommes. Mais l'abbé Burque, comme Barbeau, voit le répertoire traditionnel comme un héritage sacré en voie de contaminationil utilise en fait le terme «pollution »-par la culture populaire contemporaine. Selon Burque, les chansons traditionnelles sont menacées de disparition - et ce surtout en ville - à cause de leur trop grande « rusticité ». Dans un véritable tour de force de logique compliquée et tortueuse, il propose que les qualités mêmes qui rendent les chansons dignes d'être conservées soient la cause du dédain que les classes supérieures éprouvent en leur présence. L'homme du clergé rectifie la situation avec la hache du censeur, tandis que Barbeau opère avec la finesse du chirurgien, mais la différence n'en est peut-être au fond qu'une de style : là où Barbeau « retranche sa longue fin » à la chanson « Voici le printemps » parce que « dans son lyrisme indulgent, elle s'attarde même à des plaisirs qu'il n'est plus d'usage de glorifier en vers », admettant que ces

32. Ibid., p. 4.

33. Abbé François-Xavier Burque, Nouveau Chansonnier canadien-français, Québec, l'Imprimerie Nationale, 1921.

34. Voir, pour l'idéologie de Burque et ses objections à la méthode folklorique, Marcel Bénéteau, «Les Prêtres et religieux auteurs de chansonniers », dans Jean-Pierre Pichette (dir.), L'Apport des prêtres et religieux au patrimoine des minorités. Parcours comparés Bretagne/Canada français, Port-Acadie. Revue interdisciplinaire des études acadiennes, $\mathrm{n}^{\circ}$ 24-25-26, automne 2013-printemps 2014-automne 2014, p. 259-272. 
vers sont imagés et gracieux mais que «nous n'entendons plus ces $\operatorname{choses}^{35} »$, Burque va complètement réécrire une chanson comme «Vive la Canadienne », pièce absolument inoffensive qui, selon lui - à l'exception du premier vers - ne comporte que des « ineffabilités de débauche et d'ivrognerie ${ }^{36}$ ». Bien que les intentions soient bien différentes, les deux démarches résultent en un appauvrissement du répertoire traditionnel : les deux veulent perfectionner le répertoire ; les deux en réalité finissent par le diminuer en lui enlevant sa vitalité et son originalité.

Je propose que c'est dans ce souci de présenter la chanson dans sa forme la plus belle et la plus raffinée qu'il faut considérer - au moins en partie - l'initiative de Barbeau à créer ses fameuses « versions critiques 》. Les vers incomplets, les contradictions ou incohérences entre versions, les lacunes narratives ne sauraient laisser percevoir le potentiel artistique des chansons à des artistes raffinés. Encore ici, il faut critiquer avec soin : la démarche de Barbeau n'est pas aléatoire ; elle est basée - non sur une indignation morale comme chez l'abbé Burque - mais sur une comparaison soigneuse de nombreuses versions, de connaissances littéraires approfondies, et s'opère dans le cadre d'une tradition philologique longtemps acceptée chez les folkloristes (bien que démodée bien avant l'arrivée de Barbeau). Mais l'idée romantique de recréer une version originale - plus ancienne et plus complète que les versions recueillies dans la tradition orale - est rejetée par les ethnologues modernes justement parce qu'elle conçoit le processus folklorique comme une dégradation plutôt qu'un façonnement. Comme le souligne Marlène Belly dans son introduction au recueil de textes et mélodies de Patrice Coirault, les versions les plus anciennes comme les plus récentes « témoignent d'un état donné, à un moment et en un lieu précis, qui permet de comprendre comment l'objet a été façonné par la tradition ${ }^{37} »$.

\section{La chanson traditionnelle, sujet de recherche ethnologique ?}

Le terrain et les informateurs choisis par Barbeau et le répertoire qu'il en a extrait représentent la tradition à un moment précis, et précieux, privilégié et essentiel à la prise de conscience culturelle du Canada français. Grâce à ses enquêtes, les traces d'une ancienne tradition orale ont effectivement été sauvées de l'oubli et conservées pour les chercheurs, les artistes et le grand public. Mais il n'empêche que la démarche employée par Barbeau et sa façon de concevoir la chanson traditionnelle a eu un effet incontournable sur les chercheurs et les artistes - et je me compte dans les deux camps - qui l'ont

35. Marius Barbeau, Romancero du Canada, op. cit., p. 48.

36. Abbé François-Xavier Burque, op. cit., p. vi.

37. Marlène Belly, « Patrice Coirault, l'homme et son œuvre », dans Marlène Belly et George Delarue, Chansons françaises de tradition orale. 1900 textes et mélodies collectés par Patrice Coirault, Paris, Bibliothèque nationale de France, 2013, p. 14. 
suivi ainsi que sur la façon dont la chanson est perçue aujourd'hui. Son insistance sur l'authenticité reliée au Moyen Âge a décidément restreint le terrain d'enquête, le choix des informateurs et le champ d'intérêt. En rattachant si fermement la chanson traditionnelle à un passé glorieux et idyllique - sans mentionner rurale et illettrée - il a contribué à la mise en ghetto de la chanson traditionnelle bien séparé de la réalité contemporaine, enclave d'où on ne lui permet de sortir que dans le temps des fêtes et à d'autres moments de nostalgie privilégiée. Et enfin, l'idée du répertoire populaire a été faussée dès ses débuts en traitant la chanson traditionnelle comme un objet à conserver et à restaurer plutôt qu'un élément de la dynamique culturelle façonné par la communauté qui choisit, ou non, de le transmettre.

L'idée que seul le folkloriste peut démêler ce qui est authentique ou non a certainement influencé ceux qui ont marché dans les pas de Barbeau, à la fois dans le choix de leurs informateurs et du répertoire à recueillir. Bien que le folkloriste doive se mettre «à l'école du peuple ${ }^{38}$ », il s'agit d'un peuple défini assez étroitement ; en fait, on peut se demander s'il existe véritablement. Pour les gens, il n'y a pas de chansons « authentiques » ou « non authentiques » - il n'y a que les chansons que l'on chante. Ce que « le peuple» décide de transmettre et de valoriser ne peut qu'être « authentiquement » populaire ; même les jongleurs du Moyen Âge étaient influencés par leurs milieux, préoccupés par les soucis de l'époque, obligés de tenir compte des modes contemporaines. Mais ce n'est que très récemment que les ethnologues ont commencé à considérer les répertoires folkloriques dans leur contexte de dynamique culturelle, et non comme des produits à part conférés à une population; ce n'est qu'à la fin du $\mathrm{xx}^{\mathrm{e}}$ siècle que Fernand Harvey peut se permettre de dire que « Jusqu'ici, il semble que les ethnologues qui se sont intéressés à la culture canadienne-française aient peu réfléchi sur les conditions de son enracinement régional. La région semble avoir été considérée comme un espace d'observation plutôt que comme un lieu de production ou de reproduction de la culture traditionnelle ${ }^{39} \gg$. Ce changement de problématique transforme complètement l'approche du terrain; par exemple, dans une étude portant sur les chansons chantées aux noces dans l'est du Québec de 1920 à 1960, Anne-Marie Desdouits démontre bien que ce que les gens chantent est loin de se limiter au répertoire traditionnel français ${ }^{40}$.

38. Marius Barbeau, «En quête de connaissances [...]», op. cit.

39. Fernand Harvey, « La Problématique de la région culturelle : une piste féconde pour la recherche ? », dans Fernand Harvey (dir.), La Région culturelle. Problématique interdisciplinaire, Québec, Institut de recherche sur la culture, 1994, p. 17.

40. Voir Anne-Marie Desdouits, « Ce qu'on chantait aux noces dans l'est du Québec (19201960). Spécificité culturelle régionale ou familiale ? », dans Gérard Bouchard et Martine Ségalen (dir.), Une Langue, deux cultures, rites et symboles en France et au Québec, Québec, Presses de l'Université Laval, 1997, p. 102-121. Le répertoire analysé comporte huit catégories de chansons : chansons folkloriques françaises, chansons françaises $d u \mathrm{XIX}^{\mathrm{e}}$ siècle, variétés françaises de la première moitié 
Aujourd'hui, la chanson traditionnelle a été presque complètement évacuée des préoccupations des ethnologues. Le maintien de cette perception de la chanson comme produit plutôt que pratique l'a placée en dehors des courants scientifiques actuels et l'auraient figée en quelque sorte dans une sorte de ghetto universitaire où les ethnologues du Canada français ne s'aventurent guère ; autrefois pierre angulaire des études ethnologiques en Amérique française, la chanson a été reléguée aux oubliettes folkloriques des programmes d'études contemporaines. En ce moment, un seul cours universitaire au Canada français porte exclusivement sur la chanson traditionnelle et à peine une thèse par décennie effleure le sujet. Les chercheurs les plus importants de nos jours se trouvent décidément à l'écart des universités. Le fait que ce soient des musiciens, des artistes et des amateurs qui sont aujourd'hui les adeptes principaux de la chanson traditionnelle au Canada français - autant sur la scène que sur le terrain - suggère que ce sont eux qui sont les véritables héritiers de Marius Barbeau dans le domaine de la chanson de tradition orale. Dans le fond, c'est peut-être un développement qui aurait plu à Monsieur Barbeau.

$\mathrm{du} \mathrm{Xx}^{\mathrm{e}}$ siècle, tradition orale québécoise, autres chansons québécoises du XIX et du XX⿳⺈冂大 siècle, chansons américaines, chansons traduites en français (autres qu'américaines), opéras et opérettes (de toutes provenances). 